\title{
Is thrombin generation at the time of an acute thromboembolic episode a predictor of recurrence? The Linkoping Study on Thrombosis (LIST) - A 7-year follow-up
}

Roza Chaireti, Cecilia Jennersjö and Tomas Lindahl

\section{Linköping University Post Print}

N.B.: When citing this work, cite the original article.

Original Publication:

Roza Chaireti, Cecilia Jennersjö and Tomas Lindahl, Is thrombin generation at the time of an acute thromboembolic episode a predictor of recurrence? The Linkoping Study on Thrombosis (LIST) - A 7-year follow-up, 2013, Thrombosis Research, (131), 2, 135-139. http://dx.doi.org/10.1016/j.thromres.2012.11.015

Copyright: Elsevier http://www.elsevier.com/

Postprint available at: Linköping University Electronic Press http://urn.kb.se/resolve?urn=urn:nbn:se:liu:diva-89518 